\title{
CORRESPONDENCE
}

\section{Balancing the cognitive-behavioural approach with an analytic perspective}

Although traditional psychiatrists view delusional beliefs as being held in the face of contrary evidence normally sufficient to destroy them, Freeman \& Garety (2006) contend that they are held by evidence powerful enough to support them and their validity. They also argue for a continuum in intensity from normal to overvalued ideas to delusions, inviting consideration of similar mechanisms by which paranoid thoughts arise in the normal population and delusions in psychosis.

As a general psychiatrist using a psychoanalytic framework to relate to patients in everyday practice and aiding junior doctors through weekly psychosis workshops, I would hold a different attitude. I would distinguish the genesis of paranoid thoughts, that can occur in all of us, from delusions in major psychotic disorders.

Delusions are held in the face of contrary evidence, but we still need to know how and why they are formed. I believe that cognitive-behaviourists are correct that both emotions and reasoning come into play. What I find missing in their formulations is the integration of analytic concepts, namely the domination of the internal fantasy world over external reality in psychosis, the use of pathological projective identification in delusion formation and the importance of our countertransference feelings and sensitivities.

In the psychoanalytic model, the person with schizophrenia projects troublesome thoughts and feelings into memories stored in the mind for the purpose of disowning them, thus forming the delusion. These insights arose originally from very detailed analytic case studies by Bion (1958). I have observed that what is often disowned in the delusion is the individual's sanity.

While being in agreement with the cognitivebehaviourists that there is meaning to delusions, I believe that the primary task is to decipher their meaning through understanding the projections and subsequent rationalisations.

For example, a patient might claim that he is Prince Edward, son of Henry the Eighth. His delusion becomes understandable when it emerges that he has been assaulting his young wife, who is in a women's refuge. The delusion can be seen as his disowned sanity critical of his manically aggressive behaviour. His sanity is saying that he is acting like a son of Henry the Eighth, inventing his own rules and doing what he wants to his wives, and he wishes to disown this awareness.

I believe that $A P T^{\prime}$ 's online correspondence could provide a lively forum for reflection and debate on differences and similarities in psychological approaches towards the understanding of delusional content.

Bion, W. R. (1958) On hallucination. In Second Thoughts: Selected Papers on Psycho-analysis. Reprinted 1967: pp. 65-85. Jason Aronson.

Freeman, D. \& Garety, P. (2006) Helping patients with paranoid and suspicious thoughts: a cognitive-behavioural approach. Advances in Psychiatric Treatment, 12, 404-415.

Richard Lucas Consultant Psychiatrist/Psychoanalyst, St Ann's Hospital, London N15 IP, UK. Email: Richardnlucas1@aol.com doi: 10.1192/apt.190.2.155a

\section{CBT for psychosis}

Paranoia is a fascinating, yet challenging symptom. It still remains relatively unexplored. The extent to which it brings both subjective and objective distress or dysfunction influences its underreporting. Of more relevance to psychiatrists is how it is understood and managed in clinical practice.

Freeman \& Garety's (2006) article is of immense help in dealing with patients' paranoid symptoms, and it provides an insight into the multiple dimensions of delusions. It must not be forgotten that intelligence is largely well preserved in people with paranoia, and often responses and behaviours are consistent with the paranoid ideas. As far back as 1962, psychological interventions were offered early in the illness for these reasons.

Cognitive-behavioural therapy (CBT) helps professionals not only to clarify clinical issues, but also to fix the focus of the therapy. It becomes a guided discovery into the relevance and the understanding of the individual's experiences. A genuine curiosity and much empathy is required in a therapist. The passivity that often develops in the patient during therapy can be a hindrance, although it can also have more constructive uses. Trials have shown that good outcome can be predicted by the degree of cognitive flexibility concerning delusions. Evidence has demonstrated enduring and significant benefits of cognitive therapy applied in the acute phase of a non-affective psychotic disorder. Patients who received CBT showed significantly improved insight and fewer negative symptoms (Drury et al, 2000). Expert CBT also helps in engagement, including 